\title{
Value distribution of difference and $q$-difference polynomials
}

Nan Li and Lianzhong Yang*

${ }^{\text {*Correspondence: }}$

Izyang@sdu.edu.cn

School of Mathematics, Shandong

University, Jinan, Shandong 250100

P.R. China

\begin{abstract}
In this paper, we investigate the value distribution of difference polynomial and obtain the following result, which improves a recent result of K. Liu and L.Z. Yang: Let $f$ be a transcendental meromorphic function of finite order $\sigma, c$ be a nonzero constant, and $\alpha(z) \not \equiv 0$ be a small function of $f$, and let

$$
P(z)=a_{n} z^{n}+a_{n-1} z^{n-1}+\cdots+a_{1} z+a_{0}
$$

be a polynomial with a multiple zero. If $\lambda(1 / f)<\sigma$, then $P(f) f(z+c)-\alpha(z)$ has infinitely many zeros. We also obtain a result concerning the value distribution of $q$-difference polynomial.

MSC: Primary 30D35; secondary 39A05
\end{abstract}

Keywords: meromorphic functions; difference polynomials; uniqueness

\section{Introduction and main results}

Throughout the paper, we assume that the reader is familiar with the standard symbols and fundamental results of Nevanlinna theory as found in [1-3]. A function $f(z)$ is called the meromorphic function, if it is analytic in the complex plane except at isolated poles. For any non-constant meromorphic function $f$, we denote by $S(r, f)$ any quantity satisfying

$$
\lim _{r \rightarrow \infty} \frac{S(r, f)}{T(r, f)}=0,
$$

possibly outside of a set of finite linear measure in $\mathbb{R}^{+}$. A meromorphic function $a(z)$ is called a small function of $f(z)$ provided that $T(r, a)=S(r, f)$. As usual, we denote by $\sigma(f)$ the order of a meromorphic function $f(z)$, and denote by $\lambda(f)(\lambda(1 / f))$ the exponent of convergence of the zeros (poles) of $f(z)$.

Recently, a number of papers concerning the complex difference products and the differences analogues of Nevanlinna's theory have been published (see [4-12] for example), and many excellent results have been obtained. In 2007, Laine and Yang [10] investigated the value distribution of difference products of entire functions, and obtained the following result.

(c) $2013 \mathrm{Li}$ and Yang; licensee Springer. This is an Open Access article distributed under the terms of the Creative Commons Attribution License (http://creativecommons.org/licenses/by/2.0), which permits unrestricted use, distribution, and reproduction in any medium, provided the original work is properly cited. 
Theorem A Let $f(z)$ be a transcendental entire function of finite order, and $c$ be a nonzero complex constant. Then for $n \geq 2, f(z)^{n} f(z+c)$ assumes every non-zero value $a \in \mathbb{C}$ infinitely often.

Liu and Yang [11] improved Theorem A, and proved the next result.

Theorem B Let $f(z)$ be a transcendental entire function of finite order, and $c$ be a nonzero complex constant. Then for $n \geq 2, f(z)^{n} f(z+c)-p(z)$ has infinitely many zeros, where $p(z) \not \equiv 0$ is a polynomial in $z$.

The purpose of this paper is to investigate the value distribution of difference polynomial $P(f) f(z+c)-\alpha(z)$ and $q$-difference polynomial $P(f) f(q z)-\alpha(z)$, where $P(z)=$ $a_{n} z^{n}+a_{n-1} z^{n-1}+\cdots+a_{1} z+a_{0}$ with constant coefficients $a_{n}(\neq 0), a_{n-1}, \ldots, a_{0}$, and $\alpha(z)$ is a mall function of $f(z)$.

For the sake of simplicity, we denote by $s(P)$ and $m(P)$ the number of the simple zeros and the number of multiple zeros of a polynomial

$$
P(z)=a_{n} z^{n}+a_{n-1} z^{n-1}+\cdots+a_{1} z+a_{0}
$$

respectively.

We obtain the following result which improves Theorem A and Theorem B.

Theorem 1.1 Let $f$ be a transcendental meromorphic function of finite order $\sigma(f)=\sigma$, and $c$ be a non-zero constant, and let

$$
P(z)=a_{n} z^{n}+a_{n-1} z^{n-1}+\cdots+a_{1} z+a_{0}
$$

be a polynomial with constant coefficients $a_{n}(\neq 0), a_{n-1}, \ldots, a_{0}$ and $m(P)>0$. If $\lambda\left(\frac{1}{f}\right)<\sigma$, then $P(f) f(z+c)-\alpha(z)$ has infinitely many zeros, where $\alpha(z) \not \equiv 0$ is a small function of $f$.

Remark 1 The result of Theorem 1.1 may be false if $\alpha(z) \equiv 0$, for example, $f(z)=\frac{e^{z^{2}}}{z}$, it is obvious that $f^{2} f(z+1)$ has no zeros. The following example shows that the assumption $\lambda\left(\frac{1}{f}\right)<\sigma$ in Theorem 1.1 cannot be deleted. In fact, let $f(z)=\frac{1-e^{z}}{1+e^{z}}, c=\pi i, \alpha(z)=-1$, and $P(z)=z^{2}$. Then $\lambda\left(\frac{1}{f}\right)=\sigma(f)=1$ and $P(f) f(z+c)-\alpha(z)=\frac{2}{1+e^{z}}$ has no zeros. Also, let $f(z)=$ $i+e^{z}, c=\pi i, \alpha(z)=1$, and $P(z)=z(z-i+1)(z-i-1)$. Then $P(f) f(z+c)-\alpha(z)=-e^{4 z}$ has no zeros. This shows that the restriction in Theorem 1.1 to the multiple zero case is essential.

Considering the value distribution of $q$-differences polynomials, we obtain the following result.

Theorem 1.2 Let $f(z)$ be a transcendental entire function of zero order, and $\alpha(z) \in S(r, f)$. Suppose that $q$ is a non-zero complex constant and $n$ is an integer. If $m(P)>0$, then $P(f) f(q z)-\alpha(z)$ has infinitely many zeros. 


\section{Some lemmas}

Lemma 2.1 [6] Given two distinct complex constants $\eta_{1}, \eta_{2}$, let $f$ be a meromorphic function of finite order $\sigma$. Then, for each $\varepsilon>0$, we have

$$
m\left(r, \frac{f\left(z+\eta_{1}\right)}{f\left(z+\eta_{2}\right)}\right)=O\left(r^{\sigma-1+\varepsilon}\right) .
$$

Lemma 2.2 [6] Let $f$ be a transcendental meromorphic function of finite order $\sigma, c$ be a complex number. Then, for each $\varepsilon>0$, we have

$$
T(r, f(z+c))=T(r, f(z))+O\left(r^{\sigma-1+\varepsilon}\right)+O(\log r) .
$$

The following lemma is a revised form of Lemma 2.4.2 in [2].

Lemma 2.3 Let $f(z)$ be a transcendental meromorphic solution of

$$
f^{n} A(z, f)=B(z, f)
$$

where $A(z, f), B(z, f)$ are differential polynomials in $f$ and its derivatives with meromorphic coefficients, say $\left\{a_{\lambda} \mid \lambda \in I\right\}, n$ be a positive integer. If the total degree of $B(z, f)$ as a polynomial in $f$ and its derivatives is less than or equal to $n$, then

$$
m(r, A(z, f)) \leq \sum_{\lambda \in I} m\left(r, a_{\lambda}\right)+S(r, f) .
$$

Lemma 2.4 [12] Let $f(z)$ be a non-constant meromorphic function of finite order, $c \in \mathbb{C}$. Then

$$
\begin{array}{ll}
N\left(r, \frac{1}{f(z+c)}\right) \leq N\left(r, \frac{1}{f(z)}\right)+S(r, f), & N(r, f(z+c)) \leq N(r, f)+S(r, f), \\
\bar{N}\left(r, \frac{1}{f(z+c)}\right) \leq \bar{N}\left(r, \frac{1}{f(z)}\right)+S(r, f), & \bar{N}(r, f(z+c)) \leq \bar{N}(r, f)+S(r, f),
\end{array}
$$

outside of a possible exceptional set $E$ with finite logarithmic measure.

Lemma 2.5 [4] Letf be a non-constantzero-order meromorphic function, and $q \in \mathbb{C} \backslash\{0\}$.

Then

$$
m\left(r, \frac{f(q z)}{f(z)}\right)=o(T(r, f))
$$

on a set of logarithmic density 1.

Remark 2 For the similar reason in Theorem 1.1 in [4], we can easily deduce that

$$
m\left(r, \frac{f(z)}{f(q z)}\right)=o(T(r, f))
$$

also holds on a set of logarithmic density 1 . 
Proof Using the identity

$$
\frac{\rho^{2}-r^{2}}{\rho^{2}-2 \rho r \cos (\varphi-\theta)+r^{2}}=\operatorname{Re}\left(\frac{\rho e^{i \theta}+z}{\rho e^{i \theta}-z}\right),
$$

and let Poisson-Jensen formula with $R=\rho$, we see

$$
\begin{aligned}
\log \left|\frac{f(z)}{f(q z)}\right|= & \int_{0}^{2 \pi} \log \left|f\left(\rho e^{i \theta}\right)\right| \operatorname{Re}\left(\frac{\rho e^{i \theta}+z}{\rho e^{i \theta}-z}-\frac{\rho e^{i \theta}+q z}{\rho e^{i \theta}-q z}\right) \frac{d \theta}{2 \pi} \\
& +\sum_{\left|a_{n}\right|<\rho} \log \left|\frac{\left(z-a_{n}\right)\left(\rho^{2}-\bar{a}_{n} q z\right)}{\left(q z-a_{n}\right)\left(\rho^{2}-\bar{a}_{n} z\right)}\right| \\
& -\sum_{\left|b_{m}\right|<\rho} \log \left|\frac{\left(z-b_{m}\right)\left(\rho^{2}-\bar{b}_{m} q z\right)}{\left(q z-b_{m}\right)\left(\rho^{2}-\bar{b}_{m} z\right)}\right| \\
= & S_{1}^{\prime}(z)+S_{1}^{\prime}(z)-S_{3}^{\prime}(z),
\end{aligned}
$$

where $\left\{a_{n}\right\}$ and $\left\{b_{m}\right\}$ are the zeros and poles of $f$, respectively. Integration on the set $E:=$ $\left\{\varphi \in[0,2 \pi]:\left|\frac{f\left(r e^{i \varphi}\right)}{f\left(q r e^{i \varphi}\right)}\right| \geq 1\right\}$ gives us the proximity function,

$$
\begin{aligned}
m\left(r, \frac{f(z)}{f(q z)}\right) & =\int_{E} \log \left|\frac{f(z)}{f(q z)}\right| \frac{d \psi}{2 \pi} \\
& =\int_{E}\left(S_{1}^{\prime}\left(r e^{i \psi}\right)+S_{2}^{\prime}\left(r e^{i \psi}\right)-S_{3}^{\prime}\left(r e^{i \psi}\right)\right) \frac{d \psi}{2 \pi} \\
& \leq \int_{0}^{2 \pi}\left(\left|S_{1}^{\prime}\left(r e^{i \psi}\right)\right|+\left|S_{2}^{\prime}\left(r e^{i \psi}\right)\right|+\left|S_{3}^{\prime}\left(r e^{i \psi}\right)\right|\right) \frac{d \psi}{2 \pi} .
\end{aligned}
$$

Since $S_{i}^{\prime}=-S_{i}(i=1,2,3)$ in [4], we get $\left|S_{i}^{\prime}\right|=\left|S_{i}\right|(i=1,2,3)$.

Following the similar method in the proof of Theorem 1.1 in [4], we get the result.

Lemma 2.6 Let $f$ be a non-constant zero-order entire function, and $q \in \mathbb{C} \backslash\{0\}$. Then

$$
T(r, P(f) f(q z))=T(r, P(f) f(z))+S(r, f)
$$

on a set of logarithmic density 1.

Proof Since $f$ is an entire function of zero-order, we deduce from Lemma 2.5 that

$$
\begin{aligned}
T(r, P(f) f(q z)) & =m(r, P(f) f(q z)) \\
& \leq m(r, P(f) f(z))+m\left(r, \frac{f(q z)}{f(z)}\right) \\
& \leq m(r, P(f) f(z))+S(r, f) \\
& =T(r, P(f) f(z))+S(r, f),
\end{aligned}
$$

that is

$$
T(r, P(f) f(q z)) \leq T(r, P(f) f(z))+S(r, f) .
$$


On the other hand, using Remark 2, we get

$$
\begin{aligned}
T(r, P(f) f(z)) & =m(r, P(f) f(z)) \\
& \leq m(r, P(f) f(q z))+m\left(r, \frac{f(z)}{f(q z)}\right) \\
& \leq m(r, P(f) f(q z))+S(r, f) \\
& =T(r, P(f) f(q z))+S(r, f),
\end{aligned}
$$

that is

$$
T(r, P(f) f(z)) \leq T(r, P(f) f(q z))+S(r, f) .
$$

The assertion follows from (2.1) and (2.2).

\section{Proof of Theorem 1.1}

Let $\beta(z)$ be the canonical products of the nonzero poles of $P(f) f(z+c)-\alpha(z)$. Since $\lambda(1 / f)<$ $\sigma$ and $\alpha(z)$ is a small function of $f(z)$, we know that $\sigma(\beta)=\lambda(\beta)<\sigma(f)$. Suppose on contrary to the assertion that $P(f) f(z+c)-\alpha(z)$ has finitely many zeros. Then we have

$$
P(f) f(z+c)-\alpha(z)=R(z) e^{Q(z)} / \beta(z)
$$

where $Q(z)$ is a polynomial, and $R(z) \not \equiv 0$ is a rational function. Set $H(z)=R(z) / \beta(z)$. Then

$$
\sigma(H)<\sigma(f)=\sigma,
$$

and

$$
P(f) f(z+c)-\alpha(z)=H(z) e^{Q(z)} .
$$

Differentiating (3.2) and eliminating $e^{Q(z)}$, we obtain

$$
\begin{aligned}
& P^{\prime}(f) f^{\prime}(z) f(z+c) H(z)+P(f) f^{\prime}(z+c) H(z)-P(f) f(z+c) H^{\prime}(z)-P(f) f(z+c) Q^{\prime}(z) H(z) \\
& \quad=\alpha^{\prime}(z) H(z)-\alpha(z) H^{\prime}(z)-\alpha(z) Q^{\prime}(z) H(z) .
\end{aligned}
$$

Let $\alpha_{1}, \alpha_{2}, \ldots, \alpha_{t}$ be the distinct zeros of $P(z)$. Then

$$
P(f)=a_{n}\left(f-\alpha_{1}\right)^{n_{1}}\left(f-\alpha_{2}\right)^{n_{2}} \cdots\left(f-\alpha_{t}\right)^{n_{t}} .
$$

Substituting this into (3.3), we have

$$
\begin{aligned}
& a_{n} \prod_{j=1}^{t}\left(f-\alpha_{j}\right)^{n_{j}-1}\left\{\left(n_{1} \prod_{j \neq 1}\left(f-\alpha_{j}\right)+n_{2} \prod_{j \neq 2}\left(f-\alpha_{j}\right)+\cdots+n_{t} \prod_{j \neq t}\left(f-\alpha_{j}\right)\right)\right. \\
& \quad \times f(z+c) H(z) f^{\prime}(z)+f^{\prime}(z+c) H(z)
\end{aligned}
$$




$$
\begin{aligned}
& \left.\times \prod_{j=1}^{t}\left(f-\alpha_{j}\right)-f(z+c)\left(H^{\prime}(z)+Q^{\prime}(z) H(z)\right) \prod_{j=1}^{t}\left(f-\alpha_{j}\right)\right\} \\
= & \alpha^{\prime}(z) H(z)-\alpha(z) H^{\prime}(z)-\alpha(z) Q^{\prime}(z) H(z) .
\end{aligned}
$$

Note that $P(z)$ has at least one multiple zero, we may assume that $n_{1}>1$ without loss of generality, and we have

$$
a_{n}\left(f-\alpha_{1}\right)^{n_{1}-1} F(z, f)=\alpha^{\prime}(z) H(z)-\alpha(z) H^{\prime}(z)-\alpha(z) Q^{\prime}(z) H(z),
$$

where

$$
\begin{aligned}
F(z, f)= & \prod_{j=2}^{t}\left(f-\alpha_{j}\right)^{n_{j}-1}\left\{\left(n_{1} \prod_{j \neq 1}\left(f-\alpha_{j}\right)+n_{2} \prod_{j \neq 2}\left(f-\alpha_{j}\right)+\cdots+n_{t} \prod_{j \neq t}\left(f-\alpha_{j}\right)\right)\right. \\
& \times f(z+c) H(z) f^{\prime}(z)+f^{\prime}(z+c) H(z) \prod_{j=1}^{t}\left(f-\alpha_{j}\right) \\
& \left.-f(z+c)\left(H^{\prime}(z)+Q^{\prime}(z) H(z)\right) \prod_{j=1}^{t}\left(f-\alpha_{j}\right)\right\} .
\end{aligned}
$$

Now we distinguish two cases.

Case $1 . F(z, f) \equiv 0$. In this case, we obtain from (3.4) that

$$
\alpha^{\prime}(z) H(z)-\alpha(z) H^{\prime}(z)-\alpha(z) Q^{\prime}(z) H(z) \equiv 0 .
$$

Since $\alpha(z) \not \equiv 0$ and $H(z) \not \equiv 0$, by integrating, we have

$$
\frac{\alpha(z)}{H(z)}=k e^{Q(z)},
$$

where $k$ is a non-zero constant. From (3.2) and (3.5), we have

$$
P(f) f(z+c)=\left(\frac{1}{k}+1\right) \alpha(z)
$$

By Lemma 2.2, we have

$$
\begin{aligned}
n T(r, f(z)) & =T(r, P(f))+O(1) \\
& \leq T(r, f(z+c))+T(r, \alpha(z))+O(1) \\
& =T(r, f(z))+O\left(r^{\sigma-1+\varepsilon}\right)+S(r, f) .
\end{aligned}
$$

Since $n \geq n_{1} \geq 2$, and $f(z)$ is a transcendental, this is impossible. 
Case 2. $F(z, f) \not \equiv 0$. In this case, we set

$$
\begin{aligned}
F^{*}(z, f)= & \frac{F(z, f)}{f-\alpha_{1}} \\
= & \prod_{j=2}^{t}\left(f-\alpha_{j}\right)^{n_{j}-1}\left\{\left(n_{1} \prod_{j \neq 1}\left(f-\alpha_{j}\right)+n_{2} \prod_{j \neq 2}\left(f-\alpha_{j}\right)+\cdots+n_{t} \prod_{j \neq t}\left(f-\alpha_{j}\right)\right)\right. \\
& \times \frac{f(z+c)}{f(z)} f(z) H(z) \frac{f^{\prime}(z)}{f-\alpha_{1}}+\frac{f^{\prime}(z+c)}{f(z+c)} \frac{f(z+c)}{f(z)} f(z) H(z) \prod_{j=2}^{t}\left(f-\alpha_{j}\right) \\
& \left.-\frac{f(z+c)}{f(z)} f(z)\left(H^{\prime}(z)+Q^{\prime}(z) H(z)\right) \prod_{j=2}^{t}\left(f-\alpha_{j}\right)\right\} .
\end{aligned}
$$

Since $f(z)=\left(f(z)-\alpha_{1}\right)+\alpha_{1}$ and $f^{(k)}=\left(f-\alpha_{1}\right)^{(k)}$, we know that $F^{*}(z, f)$ is a differential polynomial of $f(z)-\alpha_{1}$ with meromorphic coefficients, and

$$
a_{n}\left(f-\alpha_{1}\right)^{n_{1}} F^{*}(z, f)=\alpha^{\prime}(z) H(z)-\alpha(z) H^{\prime}(z)-\alpha(z) Q^{\prime}(z) H(z) .
$$

By Lemma 2.3, we have

$$
\begin{aligned}
m\left(r,\left(f-\alpha_{1}\right)^{k} F^{*}(z, f)\right) \leq & 3 m\left(r, \frac{f(z+c)}{f(z)}\right)+m\left(r, \frac{f^{\prime}(z+c)}{f(z+c)}\right)+m\left(r, \frac{f^{\prime}(z)}{f-\alpha_{1}}\right) \\
& +5 T(r, H)+S(r, f)
\end{aligned}
$$

for $k=0$ and $k=1$.

Now for any given $\varepsilon(0<\varepsilon<1)$, we obtain from Lemma 2.1, Lemma 2.2 and (3.1) that

$$
\begin{aligned}
& m\left(r, \frac{f(z+c)}{f(z)}\right)=O\left(r^{\sigma-\varepsilon}\right), \quad T(r, H)=O\left(r^{\sigma-\varepsilon}\right), \\
& m\left(r, \frac{f^{\prime}(z+c)}{f(z+c)}\right)=O\left(r^{\sigma-\varepsilon}\right)+S(r, f) .
\end{aligned}
$$

The lemma of logarithmic derivative implies that

$$
m\left(r, \frac{f^{\prime}(z)}{f-\alpha_{1}}\right)=S(r, f) .
$$

It follows from (3.7) to (3.10) that

$$
\begin{aligned}
& m\left(r, F^{*}(z, f)\right)=O\left(r^{\sigma-\varepsilon}\right)+S(r, f), \\
& m\left(r,\left(f-\alpha_{1}\right) F^{*}(z, f)\right)=O\left(r^{\sigma-\varepsilon}\right)+S(r, f) .
\end{aligned}
$$

Since $\left(f-\alpha_{1}\right) F^{*}(z, f)=F(z, f)$, we obtain from the definition of $F(z, f)$ that

$$
N(r, F(z, f))=O(N(r, H(z))+N(r, f))=O\left(r^{\sigma-\varepsilon}\right)+S(r, f) .
$$


Thus,

$$
T\left(r,\left(f-\alpha_{1}\right) F^{*}(z, f)\right)=O\left(r^{\sigma-\varepsilon}\right)+S(r, f) .
$$

Note that, a zero of $f(z)-\alpha_{1}$ which is not a pole of $f(z+c)$ and $H(z)$, is a pole of $F^{\prime \prime}(z, f)$ with the multiplicity at most 1 , we know from (3.6), (3.1), Lemma 2.4 and $\lambda(1 / f)<\sigma$ that

$$
\begin{aligned}
\left(n_{1}-1\right) N\left(r, \frac{1}{f(z)-\alpha_{1}}\right) \leq & N\left(r, \frac{1}{\alpha^{\prime}(z) H(z)-\alpha(z) H^{\prime}(z)-\alpha(z) Q^{\prime}(z) H(z)}\right) \\
& +O(N(r, f(z+c)))+O(N(r, H)) \\
= & O\left(r^{\sigma-\varepsilon}\right)
\end{aligned}
$$

for the positive $\varepsilon$ sufficiently small. Hence (see the definition of $F^{*}(z, f)$ ),

$$
\begin{aligned}
N\left(r, F^{*}(z, f)\right) & =O\left(N\left(r, \frac{1}{f-\alpha_{1}}\right)+N(r, f)+N(r, H)\right) \\
& =O\left(r^{\sigma-\varepsilon}\right)+S(r, f) .
\end{aligned}
$$

It follows from (3.15) and (3.11) that

$$
T\left(r, F^{*}(z, f)\right)=O\left(r^{\sigma-\varepsilon}\right)+S(r, f)
$$

Thus, we deduce from (3.16) and (3.13) that

$$
\begin{aligned}
T(r, f(z)) & =T\left(r, f(z)-\alpha_{1}\right)+O(1)=T\left(r, \frac{\left(f-\alpha_{1}\right) F^{\prime \prime}(z, f)}{F^{*}(z, f)}\right) \\
& =O\left(r^{\sigma-\varepsilon}\right)+S(r, f) .
\end{aligned}
$$

This contradicts that $f$ is of order $\sigma$. Theorem 1.1 is proved.

\section{Proof of Theorem 1.2}

Denote $F(z)=P(f) f(q z)$. From Lemma 2.6 and the standard Valiron-Mohon'ko theorem, we deduce

$$
\begin{aligned}
T(r, F(z)) & =T(r, P(f) f(z))+S(r, f) \\
& =(n+1) T(r, f(z))+S(r, f) .
\end{aligned}
$$

Since $f$ is a entire function, then by the second main theorem and Lemma 2.5, we have

$$
\begin{aligned}
T(r, F(z)) \leq & \bar{N}(r, F(z))+\bar{N}\left(r, \frac{1}{F(z)}\right)+\bar{N}\left(r, \frac{1}{F(z)-\alpha(z)}\right)+S(r, f) \\
\leq & \bar{N}\left(r, \frac{1}{P(f)}\right)+\bar{N}\left(r, \frac{1}{f(q z)}\right)+\bar{N}\left(r, \frac{1}{F(z)-\alpha(z)}\right)+S(r, f) \\
\leq & (s(P)+m(P)) T(r, f(z))+T(r, f(q z)) \\
& +\bar{N}\left(r, \frac{1}{F(z)-\alpha(z)}\right)+S(r, f)
\end{aligned}
$$




$$
\begin{aligned}
\leq & (s(P)+m(P)) T(r, f(z))+m\left(r, \frac{f(q z)}{f(z)}\right)+m(r, f(z)) \\
& +\bar{N}\left(r, \frac{1}{F(z)-\alpha(z)}\right)+S(r, f) \\
\leq & (s(P)+m(P)+1) T(r, f(z))+\bar{N}\left(r, \frac{1}{F(z)-\alpha(z)}\right)+S(r, f),
\end{aligned}
$$

that is,

$$
\bar{N}\left(r, \frac{1}{F(z)-\alpha(z)}\right) \geq(n-s(P)-m(P)) T(r, f(z))+S(r, f(z)) .
$$

Since $f$ is a transcendental entire function with $m(P)>0$, we deduce that $P(f) f(q z)-\alpha(z)$ has infinitely many zeros.

\section{Competing interests}

The authors declare that they have no competing interests.

\section{Authors' contributions}

All authors drafted the manuscript, read and approved the final manuscript.

\section{Acknowledgements}

This work was supported by the NSF of Shandong Province, P.R. China (No. ZR2010AM030) and the NNSF of China (No. 11171013 and No. 11041005)

Received: 20 December 2012 Accepted: 25 March 2013 Published: 10 April 2013

\section{References}

1. Hayman, WK: Meromorphic Functions. Clarendon, Oxford (1964)

2. Laine, I: Nevanlinna Theory and Complex Differential Equations. de Gruyter, Berlin (1993)

3. Yi, HX, Yang, CC: Uniqueness Theory of Meromorphic Functions. Kluwer Academic, Dordrecht (2003)

4. Barnett, DC, Halburd, RG, Korhonen, RJ, Morgan, W: Nevanlinna theory for the q-difference operator and meromorphic solutions of q-difference equations. Proc. R. Soc. Edinb. A 137, 457-474 (2007)

5. Bergweiler, W, Langley, JK: Zeros of difference of meromorphic functions. Math. Proc. Camb. Philos. Soc. 142, 133-147 (2007)

6. Chiang, YM, Feng, SJ: On the Nevanlinna characteristic $f(z+\eta)$ and difference equations in the complex plane Ramanujan J. 16, 105-129 (2008)

7. Chiang, YM, Feng, SJ: On the growth of logarithmic differences, difference quotients and logarithmic derivatices of meromorphic functions. Trans. Am. Math. Soc. 361(7), 3767-3791 (2009)

8. Halburd, RG, Korhonen, RJ: Nevanlinna theory for the difference operator. Ann. Acad. Sci. Fenn. Math. 31, 463-478 (2006)

9. Halburd, RG, Korhonen, RJ: Difference analogue of the lemma on the logarithmic derivative with applications to difference equations. J. Math. Anal. Appl. 314, 477-487 (2006)

10. Laine, I, Yang, CC: Value distribution of difference polynomials. Proc. Jpn. Acad., Ser. A, Math. Sci. 83, 148-151 (2007)

11. Liu, K, Yang, LZ: Value distribution of the difference operator. Arch. Math. 92, 270-278 (2009)

12. Qi, XG, Yang, LZ, Liu, K: Uniqueness and periodicity of meromorphic functions concerning difference operator. Comput. Math. Appl. 60(6), 1739-1746 (2010)

doi:10.1186/1687-1847-2013-98

Cite this article as: Li and Yang: Value distribution of difference and q-difference polynomials. Advances in Difference Equations 20132013.98. 